\title{
The physics of the stripe quantum critical point in the superconducting cuprates
}

\author{
C. Di Castro, L. Benfatto, S. Caprara, C. Castellani, and M. Grillia \\ aINFM and Dipartimento di Fisica Università di Roma "La Sapienza" \\ 00185 Rome, Italy
}

\begin{abstract}
We elaborate on several observable consequences of the Quantum-Critical-Point scenario. In particular we show that the strong k-dependent scattering of the quasiparticles with the quasi-critical charge and spin fluctuations reproduces the main features of the low-energy spectral weights and of the observed Fermi surfaces. In the underdoped cuprates the attractive k-dependent charge scattering drives the formation of the pseudogap at the $\mathrm{M}$ points below the crossover temperature $T^{*}$. In this context we discuss models for pseudogap formation with relevant scattering in the particle-particle and particle-hole channels. The experimental consequences for the pair-fluctuation and for the pseudogap behavior are investigated.
\end{abstract}

\section{THE STRIPE QUANTUM CRITICAL POINT SCENARIO}

The non-Fermi-liquid behavior of the normalstate of the cuprates has two major features depending on the doping $(\delta)$ regimes. Specifically, (i) near optimal doping no energy scales seem to be present besides the temperature (e.g. the inplane resistivity stays linear in $T$ from just above the critical temperature $T_{c}$ ), while (ii) in the underdoped regime, new energy scales appear in the form of pseudogaps, which persist well above $T_{c}$ up to a doping-dependent crossover temperature $T^{*}$ [1]. Starting in the deeply underdoped phase, $T_{c}$ increases with increasing doping and $T^{*}$ decreases from high values of several hundreds of kelvins until it merges with $T_{c}$ near optimal doping. On the other hand, a Fermi-liquid-like behavior is observed in the overdoped materials. Correspondingly, many different physical quantities display qualitatively different behaviors in going from the under- to the optimally and to the over-doped regimes. As schematically described in Fig. 1, the subdivision of the phase diagram in three regions naturally arises from the occurrence of an instability line starting at high temperature in the deeply underdoped phase and ending at zero temperature in a quantum critical point (QCP) located near optimal doping. In this scheme the optimally doped and overdoped regimes would be related to the quantum critical (QC) and to the quantum disordered (QD) region of the QCP respectively. The two regions are separated by a crossover line $\tilde{T}(\delta)$. The underdoped regime corresponds to the (quasi)-ordered region below the instability line. However, precursor effects of the ordering could extend up to a higher temperature $T_{0}(\delta)$.

It was shown that in strongly correlated systems (e.g., in the large-U Hubbard model with an electron-phonon interaction and long-range Coulomb forces) an incommensurate chargedensity-wave instability occurs when the doping is reduced below a critical value $\delta_{c}$ [2] This tendency to order the charge arises as a compromise between the local tendency towards phase separation and the electrostatic cost to segregate charged carriers [5]. For reasonable values of the parameters this instability line starts near optimal doping at zero temperature. In the underdoped regime this charge ordering tendency occurs below a doping-dependent $T_{C D W}(\delta)$ instability line. The charge ordering strongly mixes with spin degrees of freedom and gives rise to the so-called stripe phase.

As shown in Ref. [2, 1. a crucial consequence of the stripe formation is the occurrence nearby 


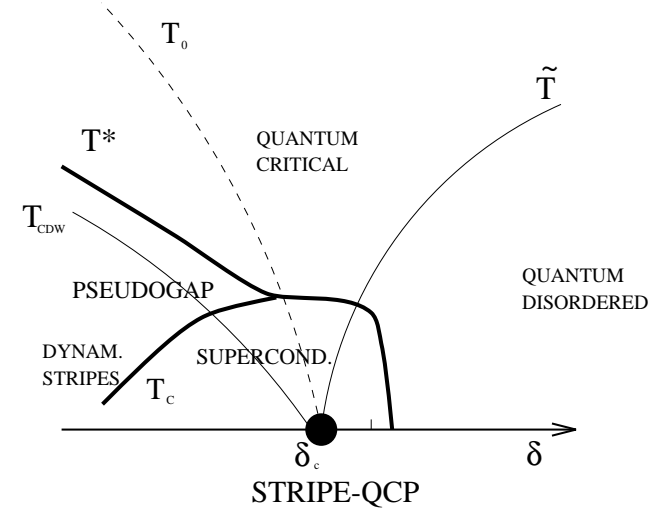

Figure 1. Schematic structure of the temperature vs. doping $\delta$ phase diagram around the StripeQCP.

the instability of a singular effective interaction, strongly dependent on momentum, doping, and temperature:

$\Gamma(\mathbf{q}, \omega) \approx \tilde{U}-\frac{V}{\kappa^{2}+\left|\mathbf{q}-\mathbf{q}_{c}\right|^{2}-i \gamma \omega}$

where $\tilde{U}$ is the residual repulsive interaction between the quasiparticles, $\gamma$ is a damping parameter, and $\mathbf{q}_{c}$ is the wavevector of the CDW instability. The crucial parameter $\kappa^{2}=\xi_{c}^{-2}$ is the inverse square of the correlation length of charge order and provides a measure of the distance from criticality. At $T=0$, in the overdoped regime, $\kappa^{2}$ is linear in the doping deviation from the critical concentration, $\kappa^{2}=a\left(\delta-\delta_{c}\right)$. In Ref. [2] the instability was found at $\delta_{c} \approx 0.2$, with $q_{c} \sim 1$. On the other hand, in the $\mathrm{QC}$ region above $\delta_{c}$, $\kappa^{2} \sim T$, according to the behavior of a Gaussian QCP. In the underdoped regime $\kappa^{2}$ vanishes approaching the instability line $T_{C D W}(\delta)$.

The occurrence of singular interactions near the QCP and near the instability line determines the physical properties of the cuprates. In particular, the non-Fermi-liquid behavior characteristic of the optimally doped materials is a signature of the QCP [2]. In the next section we report on some spectroscopic consequences of the strong scattering mediated by charge fluctuations near optimal doping. On the other hand in the overdoped region the term $\kappa^{2}=a\left(\delta-\delta_{c}\right)$ reduces the scattering and determines a region of Fermi-liquid behavior. In the underdoped compounds, when the instability line $T_{C D W}$ is approached, a singular scattering between the quasiparticles is again mediated by the charge fluctuations at wavevectors $q \approx q_{c}$. Thus the region near $T_{C D W}(\delta)$ is characterized by a strong effective interaction both in the particle-particle (p-p) and the particle-hole $(\mathrm{p}-\mathrm{h})$ channels, with a new doping-dependent energy scale. In both cases a pseudogap is an expected outcome, as it will be discussed in Section 3.

\section{SPECTRAL PROPERTIES NEAR OP- TIMAL DOPING}

The charge fluctuations couple with spin degrees of freedom since in the hole-poor regions the system is locally closer to half-filling where antiferromagnetic correlations are more pronounced. Both charge and spin fluctuations then mediate a nearly singular scattering between the quasiparticles, strongly affecting the spectral properties. In order to compare the outcomes of this scattering with the ARPES experiments, mostly performed on optimally doped Bi2212 [6], we assumed [7] a tight-binding model with the band parameters commonly accepted for this material. The exchange of QC charge fluctuations at wavevectors $\mathbf{q}_{c}= \pm(0.4 \pi,-0.4 \pi)$, and QC antiferromagnetic spin fluctuations at $\mathbf{q}_{s}=(\pi, \pi)$ was then considered within a perturbative approach. Since in general the critical wavevector is model and doping dependent, the present choice of $\mathbf{q}_{c}$ was suggested to match the experiments [6]. The resulting single-particle spectra are characterized by (i) a transfer of spectral weight from the quasiparticle peak to the incoherent shadow peaks; (ii) a redistribution of the low-energy spectral weight with a modification of the FS; (iii) a strong anisotropic suppression of spectral weight around the M points $( \pm \pi, 0)$ and $(0, \pm \pi)$. All these features have a counterpart in the experiments.

In this framework one can also investigate the bilayer structure of Bi2212 and explain the puz- 
zling absence of bonding-antibonding band splitting. According to the band calculations [8], we introduced [9] a k-dependent interplane hopping $t_{\perp}(\mathbf{k})=t_{\perp}\left|\gamma_{\mathbf{k}}\right|$ with $\gamma_{\mathbf{k}}=\frac{1}{2}\left(\cos k_{x}-\cos k_{y}\right)$. $t_{\perp}(\mathbf{k})$ is large near the $\mathrm{M}$ points only. The intraplane scattering, however, mostly reduces the quasiparticle spectral weight near the M points. Thus, within our scenario, the absence of detectable band splitting in ARPES spectra follows directly from the in-plane spectral properties.

The interaction mediated by the quasicritical fluctuations also provides an effective pairing mechanism. In this regard approaching the superconducting region from the overdoped regime, the doping and the temperature dependences of the $\kappa^{2}$ term in the $\mathrm{QD}$ and $\mathrm{QC}$ regimes give rise to a non-trivial increase of $T_{c}$ followed by a saturation around optimal doping [3]. The more involved case of the underdoped regime, with pseudogap formation will be discussed in the next section.

\section{PARTICLE-PARTICLE AND PARTI- CLE-HOLE PSEUDOGAP}

The effect of the stripe instability can be more dramatic in underdoped materials, when the system approaches the instability line at temperatures $T \sim T_{C D W}(\delta)$. In this case, near $T_{C D W}(\delta)$ the critical fluctuations at wavevectors near $\mathbf{q}_{c}$ can mediate a large effective interaction between the quasiparticles states $\mathbf{k}$ and $\mathbf{k}^{\prime}$ such that $\mathbf{k}-$ $\mathbf{k}^{\prime} \sim \mathbf{q}_{c}$. The generic outcome is that states of the Fermi surface near the M points are strongly interacting, while quasiparticles around the diagonals $(\Gamma-X$ and $\Gamma-Y$ directions $)$ are less affected. This finds a correspondence in ARPES experiments 10,11], where at $T^{*}$ the Leading Edge shift $(L E)$ starts to develop near the M points. Indeed the strong interaction near the $\mathrm{M}$ points can give rise to pairing and gaps both in the p-p and $\mathrm{p}-\mathrm{h}$ channels. Although it is quite natural that both channels contribute to the formation of the pseudogap below the crossover temperature $T^{*}$, the two limiting cases, when a single channel (either p-p or p-h) dominates the pseudogap formation, are simpler to analyze and each one of them shows relevant aspects of the physics of the cuprates.

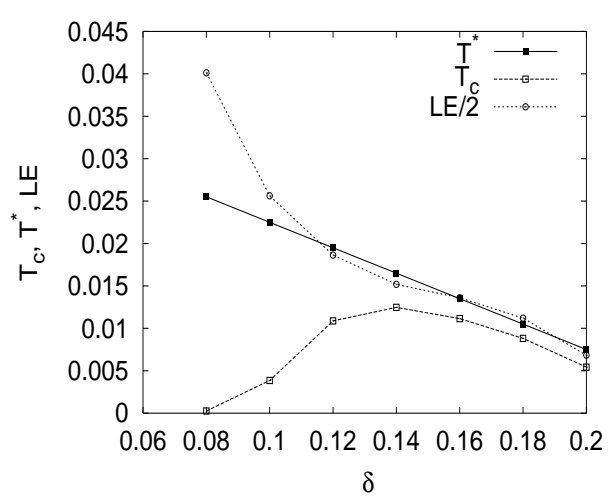

Figure 2. Doping dependence of $T^{*}, T_{c}$, and the zero-temperature $L E$. We assumed the $T^{*}(\delta)$ dependence measured in Bi2212 [11] and used $c=2.5$ and $V=90 \mathrm{meV}$ to reproduce the experimentally observed values of the $L E$ in the underdoped region and the maximum $T_{c}$.

In the first mechanism we propose, the pseudogap opens due to incoherent pairing in the p-p channel. The strong momentum dependence of the effective interaction (11) plays in this regard a crucial role in selecting the quasiparticle states which are most strongly paired. This leads to non-trivial fluctuation effects, because strongly paired states near the $\mathrm{M}$ points coexist with weakly interacting quasiparticles along the diagonals. This situation is quite different from the case described by a single superconducting order parameter $\Delta(\mathbf{k})=\Delta_{s} g(\mathbf{k})$. In the present case, indeed, the momentum dependence of the effective pairing interaction not only produces the $\mathrm{k}$ structure of $g(\mathbf{k})$, but also confers different fluctuation properties in $k$-space to the Cooper pairs depending on their strongly or weakly paired character. This physical situation has recently been described within a two-gap model 12]. In this latter framework, incoherent tightly bound Cooper pairs around the $\mathrm{M}$ points are formed at $T^{*}$, while phase coherence is established at a lower temperature $T_{c}$ by coupling to the stiffness of the weakly bound pairs near the diagonal directions. 
The scattering in the $\mathrm{p}-\mathrm{h}$ channel can provide an additional mechanism for the pseudogap formation. If this happens, the issue arises of the interplay between the preformed $\mathrm{p}$-h pseudogap and an additional BCS pairing for the weakly interacting quasiparticles. While this issue was discussed in Ref. 13] for a simple isotropic pseudogap, in Ref. 14] a specific band structure is considered, which includes a preformed k-dependent gap. The whole complication of the strong scattering around the $\mathrm{M}$ points is schematized by this preformed p-h gap $\Delta_{0}(\delta, T) \gamma_{\mathbf{k}}$, which separates the conduction and the valence band, and vanishes at the points $( \pm \pi / 2, \pm \pi / 2)$. Each band has a width $4 t \simeq 1 \mathrm{eV}, t$ being the nearestneighbor hopping. We assume $T^{*}(\delta)$ as the critical line for the preformed gap formation and take $\Delta_{0}(\delta, T)=c T^{*}(\delta) g\left(T / T^{*}(\delta)\right)$, where $c$ is a fitting parameter, $g(0)=1, g(1)=0$, and $g(x)$ interpolates smoothly between these two limits. A suitable weak pairing $V$ in the Cooper channel promotes a d-wave superconducting gap $\Delta_{s}(\delta, T) \gamma_{\mathbf{k}}$ in the low valence band of the hole doped system. The mean-field BCS critical temperature $T_{c}$ vanishes at $\delta=0$, increases with increasing doping, and reaches a maximum at $\delta_{c}$ when the chemical potential crosses the peak which individuates the pseudogap region in the density of states, and then decreases. Therefore $T^{*}$ and $T_{c}$ merge near optimum doping and the $T_{c}(\delta)$ curve has the characteristic bell-shaped form in reasonable agreement with the experiments (see Fig. 2).

The quasiparticle spectra are characterized by a $L E$, i.e. a finite minimum distance of the quasiparticle peak from the Fermi level, which persists in the normal state and is largest at the $\mathrm{M}$ points, where $L E \simeq \Delta_{0}-|\mu|$. In underdoped SC regime, the $L E$ is controlled by two parameters, as seen in experiments [15,16]. The M points are dominated by the normal-state pseudogap, whereas the nodal region are controlled by $\Delta_{s}$, which scales as $T_{c}$. In the overdoped regime the $L E \simeq \Delta_{s}$.

The above preformed gap accounts for most of the non-mean-field effects by the input of a normal-state pseudogap $\Delta_{0}(\delta, T)$. In particular, the model yields a phase diagram in good qualitative agreement with the experiments.
On the other hand, the bifurcation between $T^{*}$ and $T_{c}$ nearby optimum doping is also an outcome of the two-gap model [12]. Clearly, the two models assign a different relevance to the effect of the strong QCP effective interaction in the p-p and p-h channels, and select just one of the two channels as the most affected one. It is quite plausible that the stripe fluctuations will indeed produce non-Fermi-liquid- and non-mean-field-like effects in both channels. However, whether the results discussed above within each of the two models should cooperate to produce a better quantitative description of the cuprates, is still an open problem under investigation.

\section{REFERENCES}

1. For a recent review see, e.g. T. Timusk and B. Statt, Rep. Prog. Phys. 62, (1999) 61.

2. C. Castellani, C. Di Castro, and M. Grilli, Phys. Rev. Lett. 75, (1995) 4650.

3. A. Perali, et al., Phys. Rev. B 54, (1996) 16216.

4. C. Castellani, C. Di Castro, and M. Grilli, J. Phys. Chem. Solids 59, (1998) 1694.

5. U. Löw, et al., Phys. Rev. Lett. 72, (1994) 1918.

6. N. L. Saini, et al., Phys. Rev. Lett. 79, (1997) 3467.

7. S. Caprara, et al., Phys. Rev. B 59, (1999) 14980.

8. O. K. Andersen, et al., Phys. Rev. B49, (1994) 4145.

9. S. Caprara and M. Grilli, Journal de Physique IV (Colloques) 10, (1999) 337.

10. D. S. Marshall, et al., Phys. Rev. Lett. 76, (1996) 4871.

11. H. Ding, et al., Nature 382, (1996) 51; J. Phys. Chem. Solids 59, (1998) 1888.

12. A. Perali, et al., cond-mat/9912363, C. Castellani, et al., this proceeding.

13. P. Nozières and F. Pistolesi, Eur. Phys. J. B 10, (1999) 649.

14. L. Benfatto, S. Caprara, and C. Di Castro, preprint (2000).

15. C. Panagopoulos and T. Xiang, Phys. Rev. Lett. 81, (1998) 2336.

16. G. Deutscher, Nature 397, (1999) 410. 